\title{
www.czasopisma.pan.pl \\ Evaluation of the Mechanical Properties of Gray Cast Iron Using Electrical Resistivity Measurement
}

\author{
M. Bieroński *, O. Krasa, M. Koza, D. Książek, R. Kuś \\ Czestochowa University of Technology, \\ ul. J.H. Dąbrowskiego 69, 42-201 Częstochowa \\ *Corresponding author. E-mail address: bieronskimariusz@gmail.com
}

Received 11.03.2016; accepted in revised form 06.04.2016

\begin{abstract}
In this paper an attempt to determine the relationship between the electrical resistivity and the tensile strength and hardness of cast iron of carbon equivalent in the range from $3.93 \%$ to $4.48 \%$. Tests were performed on the gray cast iron for 12 different melts with different chemical composition. From one melt poured 6 samples. Based on the study of mechanical and electro-resistive determined variation characteristics of tensile strength, hardness and resistivity as a function of the carbon equivalent. Then, regression equations were developed as power functions describing the relationship between the resistivity of castings and their tensile strength and hardness. It was found a high level of regression equations to measuring points, particularly with regard to the relationship $\mathrm{Rm}=\mathrm{f}(\rho)$. The obtained preliminary results indicate the possibility of application of the method of the resistance to rapid diagnostic casts on the production line, when we are dealing with repeatable production, in this case non variable geometry of the product for which it has been determinated before a regression equation.
\end{abstract}

Keywords: Resistance, Grey cast iron, Tensile strength, NDT, Electrical resistivity

\section{Introduction}

In the foundry industry from many years a trend to introduce a comprehensive research systems in which the casting materials and castings are controlled with using several methods of nondestructive testing is visible. Non-destructive testing is one way to minimize the costs of risk management in the modern economy [1]. Foundry of cast iron is associated with many industries like automotive and machine. Fulfill the requirements of strength and quality, demanded by the customers is the primary aim of continuous improvement of this material [2-6]. New methods to reduce the costs while maintaining or increasing the control cycle of the produced products are still under investigation. One of such solution could be adapt the method based on the rapid measurement of the resistance of the product directly on the production line. The resistance of the casting depends on the geometry and material properties [7]. In the stabilized production conditions for a particular type of casting the resistance will be determined by the chemical composition and structure of cast iron. Changes in the cast matrix, the number and size of graphite or presence of secondary cementite, non-metallic inclusions, etc., consequently will be affected on resistance changes of the casting. In the case of casting parts of different geometries comparative analysis of the effects of structural factors can be carried out on the basis of the electrical resistivity of castings. The structure and chemical composition of the cast iron are correlated with physical properties, including mechanical properties. Grey cast iron casting 
is normally specified by its tensile strength and/or hardness. Matching the resistive characteristics of the mechanical properties can be used to approximate assessment of the quality of produced castings. Advantage of resistance measurement method is the ability to perform any number of measurements at different points of the cast including in the critical areas where the structural heterogeneity could appear. Commonly used methods of measuring tensile strength are destructive and require proper sample preparation. To carry out the classical measuring of strength, is necessary to use specialized machinery which cost is much higher than a device for measuring the resistance. Time and cost of study with using classical method causes that the search for alternative methods is reasonable, in the case of NDT, great advantage is the fact that destruction of the product is unnecessary in order to understand its mechanical properties. Possibility to verify the results of research on the finished product it is also very important for the customer and increases user confidence.

\section{Description of the approach, work methodology, materials for research, assumptions, experiments etc.}

For testing, small resistance meter - MMR-630 with very high accuracy $(0.1 \mu \Omega)$ was used. High test current for the individual sub-ranges and appropriate working modes, allow to measure also inductive objects (transformers, motors). The versatility of use this equipment is ensured by special functions, allowing to use the instrument also in the production cycle for serial measurements, for example, during control of large batches with equal size. 12 melts with different carbon equivalent were prepared from which 36 of gray cast iron samples were poured with dimensions of $123 \times 43 \times 18 \mathrm{~mm}$ for testing the resistance and 36 samples for carrying out the tensile strength test according to ISO 527-1. Samples were poured into water glass forms, hardened with $\mathrm{CO} 2$. The melting was carried out in medium frequency induction furnace with capacity of $10 \mathrm{~kg}$. In one melt were poured two forms respectively of the strength test samples and samples for the measurement of resistance. Before pouring of the metal into the mold inoculation in furnace in amount of $0.1 \%$ was introduced. Then the casts, after cooling were removed from the molds and machined to the dimensions $120 \times 40 \times 15 \mathrm{~mm}$ in order to perform the resistance measurement. For resistance measurement the 4wire method was used in order to compensate cable resistance, also bypass Kelvin probe was used - most adequate to measure the resistance of this kind of method. The measurement is based on the application of the probes on both sides of the sample at ambient temp. $20^{\circ} \mathrm{C}$, holding and reading off the displayed value. The measurement was carried out on each sample, three times, while in the table the average values of three measurement are stored. Average values were recalculated on the value of electrical resistivity with using equation;

$\rho=\frac{R S}{l}$
$\mathrm{R}$ - resistance

$\mathrm{S}-$ the transverse cross-sectional area

I - length element
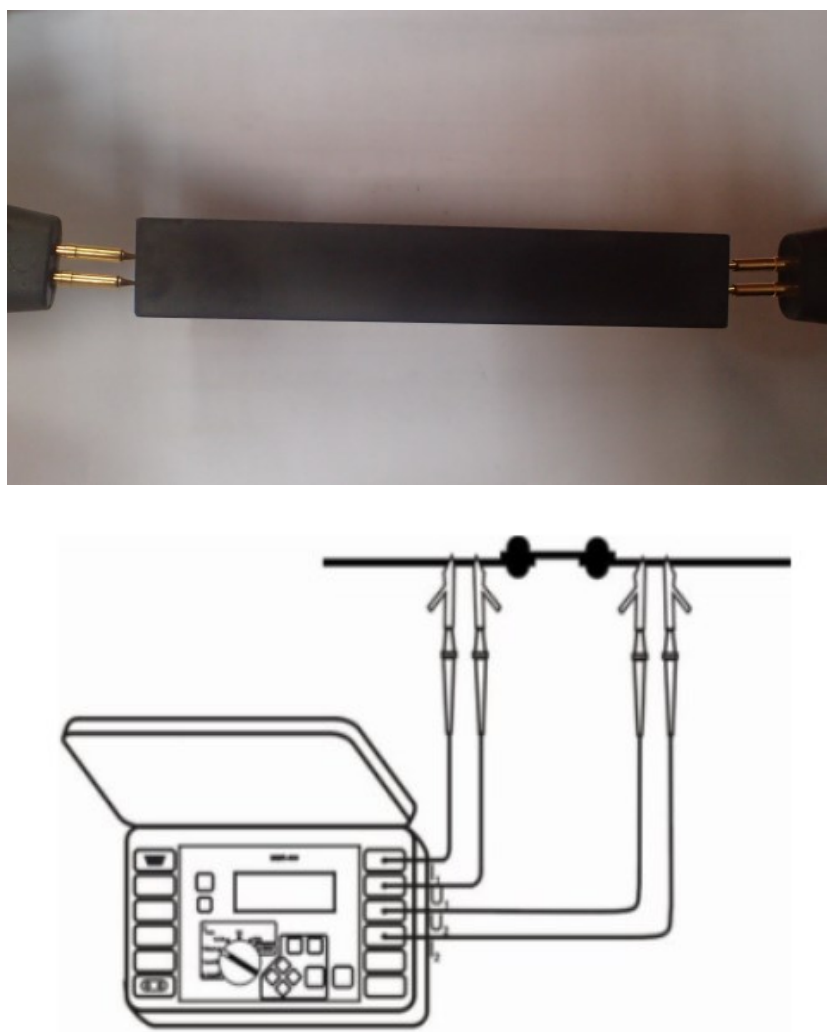

Fig.1. Measurement device of low resistance Sonel - MMR-630 with a way to measure the resistance of the samples on the side walls

Table 1.

Chemical composition of cast iron.

\begin{tabular}{cccccccc}
\hline \multirow{2}{*}{$\begin{array}{c}\text { Number } \\
\text { of iron } \\
\text { Samples }\end{array}$} & \multicolumn{6}{c}{ Chemical composition of iron \% } \\
\cline { 2 - 8 } & $\mathrm{C}$ & $\mathrm{Si}$ & $\mathrm{P}$ & $\mathrm{Mn}$ & $\mathrm{S}$ & $\mathrm{Sn}$ & $\mathrm{C}_{\mathrm{E}}$ \\
\hline 1 & 3,71 & 1,81 & 0,13 & 0,69 & 0,06 & 0,045 & 4,36 \\
\hline 2 & 3,48 & 1,87 & 0,12 & 0,66 & 0,07 & 0,043 & 4,14 \\
\hline 3 & 3,55 & 1,78 & 0,12 & 0,70 & 0,07 & 0,052 & 4,18 \\
\hline 4 & 3,22 & 2,02 & 0,12 & 0,65 & 0,06 & 0,046 & 3,93 \\
\hline 5 & 3,45 & 1,96 & 0,13 & 0,67 & 0,06 & 0,041 & 4,15 \\
\hline 6 & 3,36 & 1,92 & 0,12 & 0,73 & 0,08 & 0,038 & 4,04 \\
\hline 7 & 3,79 & 1,93 & 0,13 & 0,70 & 0,08 & 0,042 & 4,48 \\
\hline 8 & 3,65 & 1,69 & 0,13 & 0,72 & 0,07 & 0,042 & 4,26 \\
\hline 9 & 3,39 & 1,77 & 0,12 & 0,76 & 0,09 & 0,045 & 4,02 \\
\hline 10 & 3,33 & 2,00 & 0,12 & 0,77 & 0,10 & 0,042 & 4,04 \\
\hline 11 & 3,76 & 1,49 & 0,13 & 0,66 & 0,07 & 0,041 & 4,30 \\
\hline 12 & 3,68 & 1,76 & 0,13 & 0,67 & 0,06 & 0,039 & 4,31 \\
\hline
\end{tabular}

Where; 
Table 2.

The results of the tensile strength, HB resistance and electrical resistivity cast samples.

\begin{tabular}{ccccc}
\hline $\begin{array}{c}\text { Number } \\
\text { of iron } \\
\text { Samples }\end{array}$ & $\begin{array}{c}\text { HB } \\
\text { Brin- } \\
\text { ella }\end{array}$ & $\begin{array}{c}\text { Tensile } \\
\text { strength } \\
\text { Rm } \\
(\mathrm{Nmm} 2)\end{array}$ & $\begin{array}{c}\text { Resistance } \\
\mathrm{R}(\mu \Omega)\end{array}$ & $\begin{array}{c}\text { Electrical } \\
\text { resistivity } \\
\rho\left(\mathrm{m}^{*} \mu \Omega\right)\end{array}$ \\
\hline 1 & 185 & 177 & 151 & 0,755 \\
\hline 2 & 197 & 241 & 130 & 0,650 \\
\hline 3 & 205 & 224 & 134 & 0,670 \\
\hline 4 & 221 & 294 & 119 & 0,595 \\
\hline 5 & 208 & 228 & 131 & 0,655 \\
\hline 6 & 202 & 268 & 121 & 0,605 \\
\hline 7 & 168 & 146 & 164 & 0,820 \\
\hline 8 & 205 & 220 & 130 & 0,650 \\
\hline 9 & 221 & 259 & 122 & 0,610 \\
\hline 10 & 218 & 269 & 117 & 0,585 \\
\hline 11 & 201 & 215 & 139 & 0,695 \\
\hline 12 & 209 & 210 & 134 & 0,670 \\
\hline & & & &
\end{tabular}

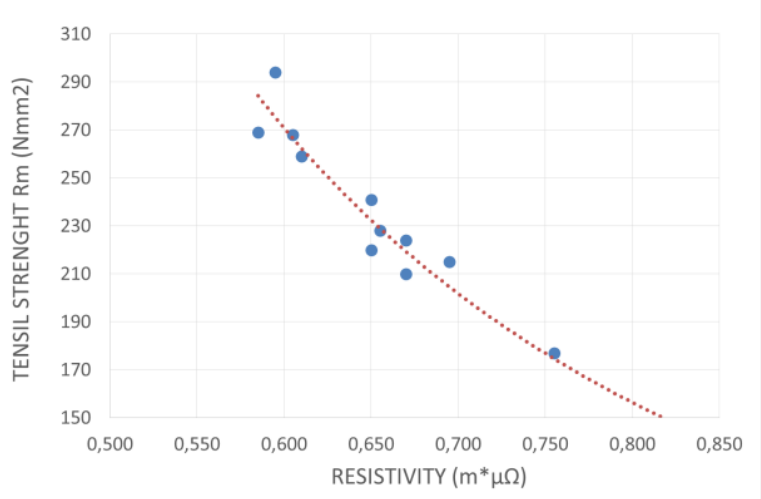

Fig. 2. A plot of tensile strength $(\mathrm{Rm})$ versus electrical

\section{Description of achieved results}

Based on the study, relationship between electrical resistivity and hardness and tensile strength was determined. The resulting regression equation for the hardness is $\mathrm{R}^{2}=0,83$, and for tensile strength $\mathrm{R}^{2}=0,96$. The regression equation are applicable to a range of the carbon equivalent in the range of $\mathrm{CE}=3.93-4.48$ and pieces measuring $120 \times 40 \times 15 \mathrm{~mm}$;

$$
H B=150,79 * p^{-714}
$$

$$
R m=102,13 * p^{-1,909}
$$

Where;

$\mathrm{CE}$ - carbon equvalent

$\mathrm{p}$ - resistivity

$\mathrm{Rm}$ - tensile strenght

Dependence of mechanical properties of cast-iron is shown on Figs. 2-5 and structure examples of the investigated cast-iron on Figs. 6 and 7. resistivity $(\rho)$

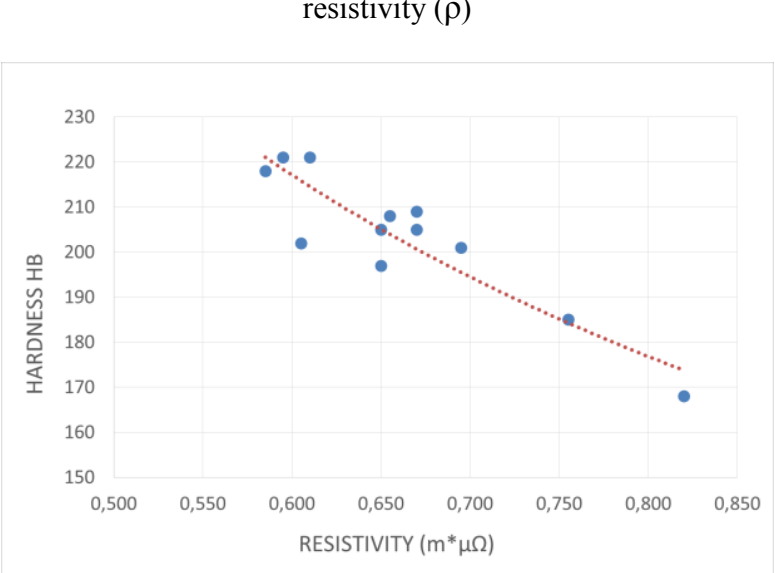

Fig. 3. A plot of Brinell hardness (HB) versus electrical resistivity $(\rho)$

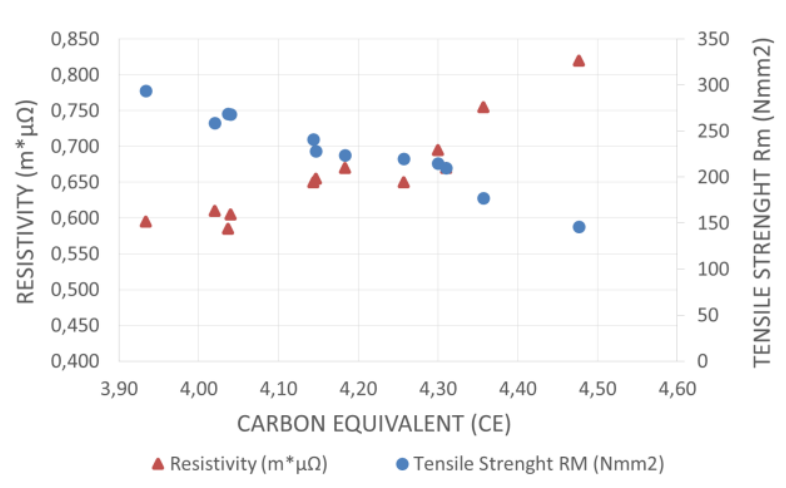

Fig. 4. A plot of $\mathrm{Rm}=\mathrm{f}(\mathrm{CE})$ and $\rho=\mathrm{f}(\mathrm{CE})$ versus carbon equivalent 


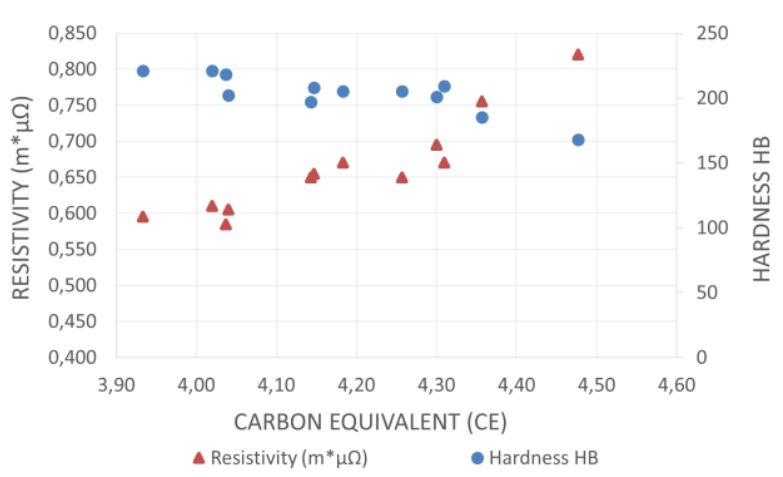

Fig. 5. A plot of $\mathrm{HB}=\mathrm{f}(\mathrm{CE})$ and $\rho=\mathrm{f}(\mathrm{CE})$ versus carbon equivalent

With increasing CE equivalent marking, resistivity of iron is also increasing, the trend is reversed in relation to the changes in tensile strength, for each $0.1 \%$ of increase in $\mathrm{CE}$, resistivity is increase by 0.05 , and the resistivity range from 0.6 to 0.7 is equivalent of $100 \mathrm{Rm}$ range. Samples were etched with $4 \%$ nital, x500 magnification. The sample of the melt no. 4 has a smaller amount of graphite flake with a pearlite matrix with fine ferrite precipitates to $5 \%$ and $3 \%$ of cementite, there is also a greater dispersion of cementite platelets at pearlite grains.

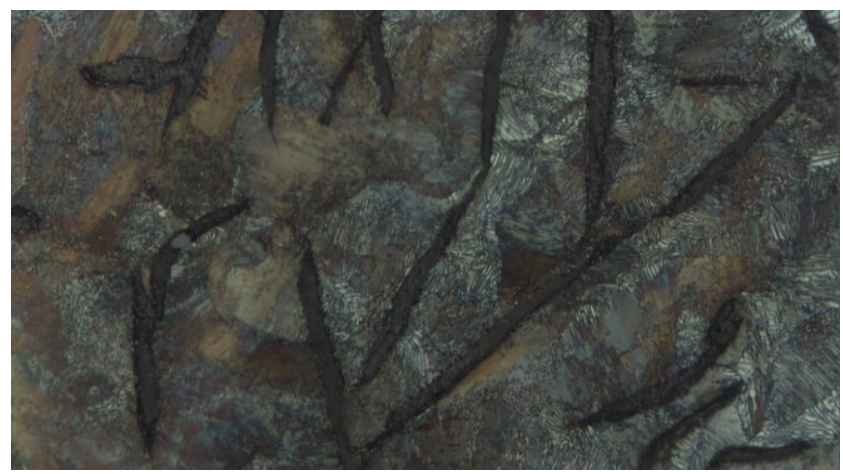

Fig. 6. The structure of cast iron samples no 4. 500x

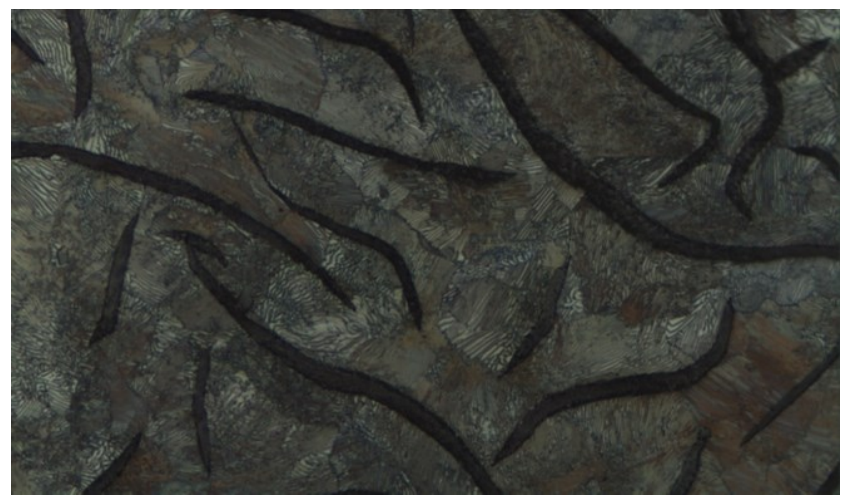

Fig. 7. The structure of cast iron samples no $1.500 \mathrm{x}$
Small amount of fine ferrite precipitates uniformly distributed on the samples surface is visible. Sample of the melt no. 1 has a higher number of graphite flake with a pearlite matrix with fine ferrite precipitates to $5 \%$ and $1 \%$ of cementite. Small amount of fine ferrite precipitates uniformly distributed on the samples surface is visible.

\section{Conclusion}

Based on the obtained results it should be stated that, electrical resistivity at constant dimensions is strongly correlated with tensile strength parameter. An interesting fact is that the presented correlation can be also find in the tables in publication „Precise wire resistive alloy $\mathrm{Cu}-\mathrm{Ni}-\mathrm{Mn} \mathrm{I}$ Ni-Cr-Al-Si” [4-5]. Correlation in researched ranges, with high accuracy, the tensile strength values could be determined and converted, based on electrical resistivity measurement. Repeatability of the measurement methodology deserves for particular attention, which affects the accuracy of the test results. Resistance measurement is particularly dedicated to serial production, such as: engine blocks, ballasts, etc., because it gives the opportunity to forecast the measurement for fixed geometry. The method is easy to use, does not require a special permission and the cost of the tooling is approximately 15 times lower than for standard method. Based on the above conclusions it can be stated that the continuation of testing the resistance or electrical resistivity of cast iron gives a great opportunity to learn about the correlation also for many other properties of the material and materials used for its production, for example: determining the quality of carburizers as coke or carburizers graphitizing after appropriate preparation for testing.

\section{References}

[1] Orłowicz, W. (2001). Ultrasonic quality inspection of iron castings. Archives of Foundry. 1(1), 211-226 (in Polish)

[2] Janerka, K. (2010). Carburizing liquid iron alloys Gliwice: Wydawnictwo Politechniki Śląskiej. (in Polisch)

[3] Dudyk, M. (2000). Application of the silieon carbide for the melting of grey cast iron in the cupola. Solidification of Metais and Alloys. 2(42), 87-93. (in Polish).

[4] Kamionka, T, \& Galanty, M. (1992). Properties of the resistive wire, $\mathrm{NiCr}$ AlSi alloy according to the method of preparing. Rudy i metale niezależne. 37, 220-225. (in Polish).

[5] Kamionka, T. \& Galanty, M. (1993). Precise wire resistive alloy $\mathrm{Cu}-\mathrm{Ni}-\mathrm{Mn}$ i Ni-Cr-Al-Si. Materiaty Elektroniczne T. 21(1). (in Polish).

[6] Lenard. W. (1978). Study the tensile strength of gray cast iron in casting ultrasonic. Przeglad Odlewnictwa. 2, 37-39. (in Polish).

[7] Pittaccio, S. \& Garavaglia, L. (2014). Electric resistance monitoring as a method for controlling shape memory alloy characteristics during shape-setting treatments in the furnace. Materials Science \& Engineering. A 599. 92-10. 